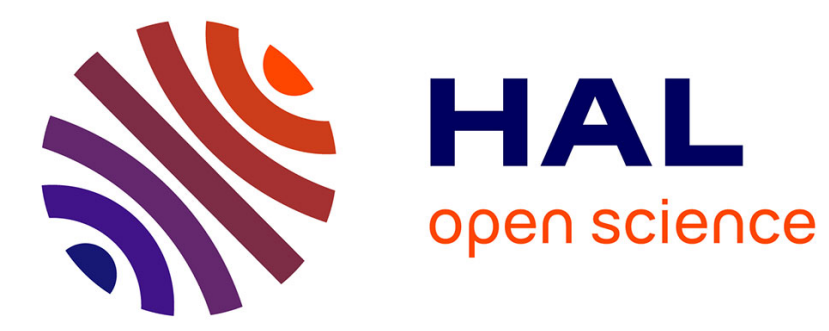

\title{
On the convective-absolute nature of river bedform instabilities
}

Riccardo Vesipa, Carlo Camporeale, Luca Ridolfi, Jean Marc Chomaz

\section{To cite this version:}

Riccardo Vesipa, Carlo Camporeale, Luca Ridolfi, Jean Marc Chomaz. On the convective-absolute nature of river bedform instabilities. Physics of Fluids, 2014, 26 (12), pp.124104. 10.1063/1.4902901 . hal-01114977

\section{HAL Id: hal-01114977 \\ https://hal-polytechnique.archives-ouvertes.fr/hal-01114977}

Submitted on 10 Feb 2015

HAL is a multi-disciplinary open access archive for the deposit and dissemination of scientific research documents, whether they are published or not. The documents may come from teaching and research institutions in France or abroad, or from public or private research centers.
L'archive ouverte pluridisciplinaire HAL, est destinée au dépôt et à la diffusion de documents scientifiques de niveau recherche, publiés ou non, émanant des établissements d'enseignement et de recherche français ou étrangers, des laboratoires publics ou privés. 


\section{AIP $\left.\right|_{\text {Fyids }} ^{\text {Physe of }}$}

\section{On the convective-absolute nature of river bedform instabilities}

Riccardo Vesipa, Carlo Camporeale, Luca Ridolfi, and Jean Marc Chomaz

Citation: Physics of Fluids (1994-present) 26, 124104 (2014); doi: 10.1063/1.4902901

View online: http://dx.doi.org/10.1063/1.4902901

View Table of Contents: http://scitation.aip.org/content/aip/journal/pof2/26/12?ver=pdfcov

Published by the AIP Publishing

\section{Articles you may be interested in}

Direct numerical simulation of transitional mixed convection flows: Viscous and inviscid instability mechanisms

Phys. Fluids 25, 094102 (2013); 10.1063/1.4821149

Modal versus nonmodal linear stability analysis of river dunes

Phys. Fluids 23, 104102 (2011); 10.1063/1.3644673

Absolute and convective instability of cylindrical Couette flow with axial and radial flows

Phys. Fluids 21, 104102 (2009); 10.1063/1.3243976

The physics of evaporative and convective instabilities in bilayer systems: Linear theory Phys. Fluids 16, 4644 (2004); 10.1063/1.1812671

Nonlinear convective/absolute instabilities in parallel two-dimensional wakes

Phys. Fluids 10, 2724 (1998); 10.1063/1.869796

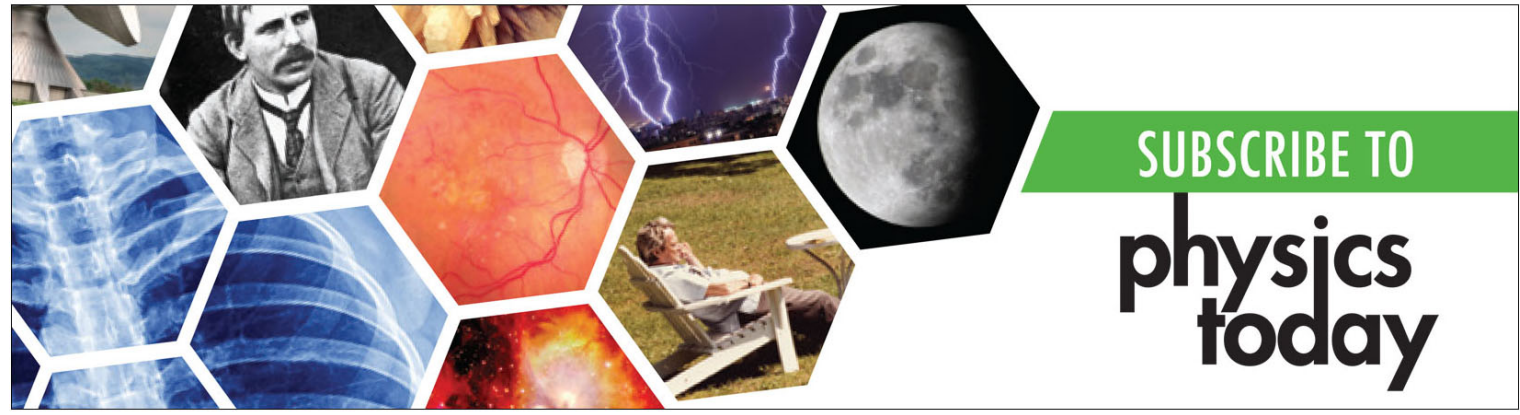




\title{
On the convective-absolute nature of river bedform instabilities
}

\author{
Riccardo Vesipa, ${ }^{1}$ Carlo Camporeale,${ }^{1}$ Luca Ridolfi, ${ }^{1}$ \\ and Jean Marc Chomaz ${ }^{2}$ \\ ${ }^{1}$ Department of Environmental, Land and Infrastructure Engineering, Politecnico di Torino, \\ C.so Duca degli Abruzzi 24, 10129 Torino (TO), Italy \\ ${ }^{2}$ Laboratoire d'Hydrodynamique, Ecole Polytechnique, 91128, Palaiseau, France
}

(Received 8 August 2014; accepted 15 November 2014; published online 9 December 2014)

\begin{abstract}
River dunes and antidunes are induced by the morphological instability of streamsediment boundary. Such bedforms raise a number of subtle theoretical questions and are crucial for many engineering and environmental problems. Despite their importance, the absolute/convective nature of the instability has never been addressed. The present work fills this gap as we demonstrate, by the cusp map method, that dune instability is convective for all values of the physical control parameters, while the antidune instability exhibits both behaviors. These theoretical predictions explain some previous experimental and numerical observations and are important to correctly plan flume experiments, numerical simulations, paleo-hydraulic reconstructions, and river works. @ 2014 AIP Publishing LLC. [http://dx.doi.org/10.1063/1.4902901]
\end{abstract}

\section{INTRODUCTION}

Morphological stability analysis of a loose granular bed interacting with a turbulent freesurface stream is a long standing problem in fluid mechanics, and it shares many affinities with open shear flow instabilities. A cross-disciplinary scientific community is repeatedly intrigued by a number of issues in sediment bed form dynamics, from the uppermost theoretical ones (transient behavior, ${ }^{1}$ role of $3 \mathrm{D}$ effects $^{2}$ ) to important questions concerning the anthropic activity in rivers and several environmental aspects. In this paper, dunes and antidunes are considered. They are micro-scale river bedforms; periodic patterns that scale with the water depth and that induce a free surface perturbation. When the free surface is out of phase with respect to the bedforms, dunes occur. This usually happens in subcritical conditions ( $F<1$, where $F$ is the stream Froude number) and leads to downstream migrating bedforms. If the free surface is in phase with respect to the bedforms, instead, antidunes arise. This is common for supercritical streams $(F>1)$ and leads to upstream migrating bedforms, even if some recent analyses have shown that in subcritical streams, downstream migrating antidunes may occur. ${ }^{3}$

In spite of the incredible theoretical, numerical, and experimental efforts, devoted to the analysis of dune inception, growth, and nonlinear behavior, the absolute/convective nature of dunes and antidunes remains unexplored. This topic has been proven to provide key information on unstable open flow hydrodynamic systems, ${ }^{4}$ as well as on meso- and macro-scale morphodynamic systems, such as bars ${ }^{5}$ and meanders. ${ }^{6}$ The assessment of the absolute/convective nature of the instability is a fundamental analysis (carried out at the linear level) that adds new key information with respect to those obtainable only from a stability analysis (typical results of a stability analysis are the growth rate, the phase velocity, the direction of migration, the most amplified wavenumber of the perturbation). Physically speaking, a system is convectively unstable if the response to an impulsive perturbation increases in time but migrates and decays to zero at all spatial locations, or absolutely unstable if the response grows exponentially in time at all spatial locations. To unveil the nature of instability of micro-scale bedforms allows flume experiments and numerical simulations to be correctly set and the obtained results to be correctly interpreted and understood. Furthermore, such an investigation is fundamental for the correct interpretation of paleohydraulic reconstruction ${ }^{7}$ and can play a key role in the design of river infrastructures. 
In order to discriminate between these two kinds of instabilities, one needs to determine if the wave with the zero group velocity is growing (absolute) or decaying (convective). This is an easy task only in a limited number of cases, where the dispersion relation is provided analytically. Unfortunately, this is not the case for micro-scale bedforms which require a refined modeling of the flow field along the non-homogeneous vertical direction. This drawback is the reason for the gap in the literature, if compared to the case of other morphodynamic instabilities (e.g., bars ${ }^{5}$ and meanders ${ }^{6}$ ) which are shallow water-driven and do not require to solve for the eigenfunction in the vertical direction. In order to circumvent this difficulty, we will apply the so-called cusp map method described by Kupfer et $_{\text {al. }}{ }^{8}$ to the present problem.

Over the last 50 years, since the seminal work of Kennedy, ${ }^{9}$ progresses have been made in the theoretical study of dunes and antidunes, ${ }^{10-14}$ and it is now understood that bedforms are the result of a morphological linear/nonlinear instability of the stream-sediment interface. ${ }^{15}$ Very refined and reliable models ${ }^{12,14,16,17}$ can capture the key physical mechanisms of bedforms inception and provide very good matching with experimental observations from laboratory and field data. Nevertheless, some issues are still debated, and a universally recognized and fully accepted complete mathematical model describing all phases of the inception, the growth, and the nonlinear evolution of dunes is still a matter of current research. The main reason for this difficulty is the complexity of the sediment transport processes that ultimately drive the morphological evolution of the bottom. As a matter of fact, complex mechanisms such as the entrainment of particles in the sediment flux, the collision between particles, and the fluid-particle interactions make the precise modeling of the sediment transport extremely difficult and challenging. Over the last decade, in particular, two aspects have attracted researchers' interest.

The first aspect is the particle inertia. In non-uniform conditions, in fact, sediment particles require a non-negligible time for adapting to changes of the velocity of the surrounding fluid. This implies that the adaptation of the sediment discharge to changing in the shear stress is not instantaneous, rather, it takes place over a finite distance called saturation length. This saturation length was found to be a key parameter for the growth of regular patterns, through the selection of a dominant wavelength, in sub-aqueous bedforms. ${ }^{14}$

The second aspect is the transfer of momentum from the liquid phase to the sediment particles occurring in the bedload layer. The bedload layer is the thin layer in which all moving grains that are rolling, sliding, or saltating are concentrated. The energy transfer from fluid to particles alters the fluid stress profile inside the bedload layer. ${ }^{18,19}$ This implies that the fluid shear stress responsible for the sediment discharge, and therefore for the morphological evolution of the bottom, is the stress acting at the top of the bedload layer rather than the stress acting at the bottom.

Different assumptions in the modeling of the sediment transport allow the identification of different physical mechanisms driving the bedform evolution, especially for what concerns the origin of dunes. On one side, sediment inertia is considered to be the key factor that allows for the inception of regular patterns of ripples, while Fourriere et al. ${ }^{14}$ and Charru et al. ${ }^{20}$ suggested that dunes arise from the nonlinear coarsening of this early ripple pattern. On the other side, if the shear stress responsible for the sediment transport is evaluated at the top of the bedload layer, both dunes and ripples arise as primary instability. ${ }^{12,17}$

It is not the aim of the paper to review the details of the two different approaches (as already done by two of the authors ${ }^{21}$ ), and in the following, bedforms will be modeled through the Colombini's ${ }^{12}$ approach. We have adopted the aforementioned model for its physical strength and for the very good performances in testing against experimental data. In particular, (i) the main assumption, i.e., the shear stress responsible of the motion of sediment particles is not the shear stress acting at the water-sediment interface but the shear stress acting at the top of the bedload layer finds its theoretical justification and experimental validation in several works; ${ }^{18,19}$ (ii) the match with the fundamental set of experimental benchmark data of Guy et al. ${ }^{22}$ is very good; (iii) ripple instability can be predicted as well, provided that smooth and transitional flow regimes are considered in the mathematical formulation of the model ${ }^{17}$ (iv) the wavelength selection occurring in the transitory period of evolution from flat bed to fully developed bedforms can be attributable to linear interactions among non-orthogonal modes ${ }^{21}$ while, to the authors knowledge, no theoretical proof has ever clarified the role of nonlinearities in the mechanism of ripple coarsening; (v) the outcomes from the 
nonlinear analysis ${ }^{23}$ of the same model, as well as from the inclusion of $3 \mathrm{D}$ effects, ${ }^{2}$ provide very good matching with experimental data.

\section{MODEL AND METHODS}

A turbulent incompressible open channel flow on erodible cohesionless sand bed is considered. The (homogeneous) sediment is characterized by the relative grain diameter, $d_{s}=d_{s}^{*} / D_{0}\left(D_{0}\right.$ is the mean flow depth and $d_{s}^{*}$ the dimensional grain diameter), and the sediment-to-water density ratio, $R=\rho_{s} / \rho$. The friction velocity $u_{\tau}$ and $D_{0}$ are used for the scaling of all quantities and hydrodynamics is considered adiabatically slaved to the bed topography evolution (the quasi-steady approximation). The right-handed Cartesian frame $\{x, y, z\}$ reported in figure 1 is adopted, indicating the dimensionless stream-wise, span-wise, and normal-to-the bed coordinates, respectively. The stream is bounded by the free surface $z=H(x, y, t)$ and by the fluid-sediment interface $z=\Theta(x, y, t)$. Suspended and washed loads are neglected, so that the overall sediment transport takes place in form of bedload only, wholly contained in the so-called saltation layer $(0<z-\Theta<\delta)$, where $\delta$ is the thickness of the saltation layer.

In order to address the fundamental dynamics of bed forms instabilities, and to ease the comparison with experiments ${ }^{24}$ and simulations, ${ }^{25}$ we will focus on $2 \mathrm{D}$ patterns $(\partial / \partial y=0)$. Colombini et $a .^{2}$ have recently showed that three dimensional effects become crucial when either the channel width/depth ratio or the grain size to depth ratio is very large. Also, most of laboratory data obtained in narrow flumes show essentially $2 \mathrm{D}$ structures ${ }^{26}$ and the early development of the bed perturbations is preferentially observed as two-dimensional. ${ }^{27}$ Since bed forms evolve on a slow time scale, the water layer is quasi steady and governed by the dimensionless Reynolds and continuity equations

$$
\begin{gathered}
\mathbf{u} \cdot \boldsymbol{\nabla} \mathbf{u}+\nabla P+\boldsymbol{\nabla} \cdot \mathbf{T}+\mathbf{f}=0, \\
\boldsymbol{\nabla} \cdot \mathbf{u}=0,
\end{gathered}
$$

where $\mathbf{u}=\{u, w\}$ is the velocity vector, $P$ the pressure, $\mathbf{T}$ the deviatoric turbulent stress tensor, $\mathbf{f}=\{-1,1 / \tan \beta\}$ the body force vector, and $\beta$ the channel mean inclination with respect to the horizontal. The open-channel hydrodynamic problem is completed by suitable boundary conditions at the free surface $(z=\Theta+D), \mathbf{u} \cdot \mathbf{n}=\mathbf{n} \cdot \mathbf{T}=\mathbf{0}$, and at the bottom $(z=D), \mathbf{u}=\mathbf{0}$ (unit vector $\mathbf{n}$ is defined in Fig. 1).

For analytical and numerical convenience, we apply a Prandtl-like mapping to the above equations which rectangularizes the domain: $z \in[\Theta, \Theta+D] \rightarrow \zeta=(z-\Theta) / D \in[0,1]$. In order to evaluate the deviatoric stress tensor in (1a), we adopt a Boussinesq-type turbulence closure model, i.e., $\mathbf{T}=-2 v_{T} \mathbf{S}$, where $\mathbf{S}$ is the deformation rate tensor and the eddy viscosity is evaluated according to a mixing length model: ${ }^{28} v_{T}=l^{2} D^{2}[2 \mathbf{S} \cdot \mathbf{S}]^{1 / 2}$. Empirical data ${ }^{29}$ provide $l=\kappa$

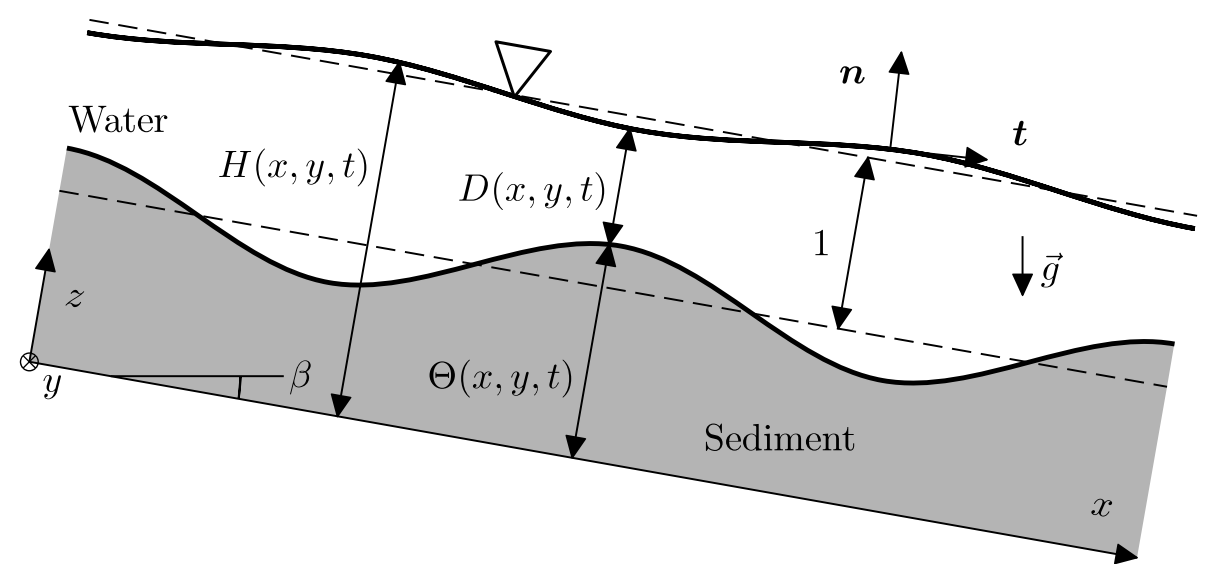

FIG. 1. Sketch of the physical problem. Unperturbed, flat bed (dashed line) and perturbed bed (thick continuous line). 
$(1-\zeta)^{1 / 2}\left(\zeta+\zeta_{r}\right)$, where $\kappa$ is von Karman constant and $\zeta_{r}=d_{s} / 12$ is the roughness displacement ${ }^{12}$ here assumed to be linked to the grain diameter.

Finally, stream dynamics is coupled with the bed evolution by the sediment mass balance, namely, the Exner's equation ${ }^{30}$

$$
\begin{gathered}
\frac{\partial \Theta}{\partial t}+Q_{0} \frac{\partial \Phi}{\partial x}=0, \\
Q_{0}=\frac{C d_{s} \sqrt{(R-1) d_{s}}}{F(1-\phi)}, \\
F=\frac{C u_{\tau}}{\sqrt{g D_{0}}},
\end{gathered}
$$

where $\phi$ is the sediment porosity, $C=(1 / \kappa) \ln \left(4.43 / d_{s}\right)$ is a friction coefficient, $g$ is the gravity acceleration, and $\Phi$ is the dimensionless sediment transport rate.

The latter term is computed as $\Phi=r\left(\Phi_{e q}\right)$, where $\Phi_{e q}$ is the sediment flux rate evaluated under equilibrium (unperturbed) conditions while the function $r$ accounts for local variations induced by the bottom perturbation. After suitable scaling, most of the known formulas for $\Phi_{e q}$ can be recast as ${ }^{21} \Phi_{e q} \sim\left(\theta_{\delta}-\theta_{c}\right)^{\sigma}$, where $\sigma$ is an empirical coefficient while $\theta=\tau^{*} /\left[\left(\rho_{s}-\rho\right) g d_{s}^{*}\right]$ refers to the Shields dimensionless shear stress, where $\tau^{*}$ is the dimensional shear stress exerted by the fluid (the meaning of subscripts are explained in the following). Incidentally, we recall that the hydrodynamic-to-morphodynamic timescale ratio $\left(\sim Q_{0}\right)$ is usually $O\left(10^{-2}\right)$ or less, which justifies the choice of the quasi-steady approximation.

It is customary to complement the above model with three further specifications. First, Colombini $^{12}$ demonstrated that the Shields stress responsible for the motion of the sediment particle has to be accounted for at the top of the saltation layer, ${ }^{18,19}$ namely, $\theta_{\delta}:=\left.\theta\right|_{\zeta=\delta}$. This choice is crucial to model the occurrence of both dunes and antidunes correctly. Second, the slope-dependent threshold value $\theta_{c}$, below which the flow is not able to move sediment particles, is set to $\theta_{c}$ $=\theta_{t h}\left[1-\left(\tan \beta-\Theta_{, x}\right) / \mu\right]$, where comma notation refers to the partial derivative, $\theta_{t h}$ is the Shields stress threshold for sediment transport in a nearly horizontal channel and $\mu=0.10$ is a dimensionless constant. ${ }^{31}$ Third, the inertial adaptation of the sediment particles to the topography-induced changes in the shear stress is taken into account according to a flow saturation approach ${ }^{32}$ which imposes $L_{s} \Phi_{, x}=\Phi_{e q}-\Phi$. This is equivalent to set $r=\Phi_{e q}\left[1+L_{s} \partial_{x}\right]^{-1}$. The saturation length, $L_{s}$, is the typical length required by the sediment flow to reach its equilibrium value $\Phi_{e q}{ }^{14}$

Following the outcomes by Camporeale, ${ }^{21}$ a Meyer-Peter-Muller-like formulation $(\sigma=3 / 2)$ will be adopted in the sequel, with the setting $L_{s}=0$ and $\delta=l_{b} d_{s}$, where $l_{b}=1+1.3\left[\left(\left.\tau\right|_{\zeta=0}\right.\right.$ $\left.\left.-\tau_{c}\right) / \tau_{c}\right]^{0.55}$ as suggested by Colombini ${ }^{12}$ on the basis of experimental data. ${ }^{33,34}$ This choice was proved to be the minimum requirement for a correct prediction of dunes and antidunes instability in the parameter space. In the present case of sub-aqueous bedforms, further efforts to improve the setting of the saturation length or the saltation layer thickness are not rewarded by a better agreement with the experimental benchmark.

We now perturb the vector $\mathbf{g}=\{u, w, P, \Theta, D\}$ as $\mathbf{g}=\mathbf{G}+\epsilon \hat{\mathbf{g}} \exp [\mathrm{i}(k x+\omega t)]+$ c.c., where $\mathbf{G}=\left\{u_{0}(\zeta), 0, p_{0}(\zeta), 0,1\right\}$ is the (streamwise independent) basic state, $\hat{\mathbf{g}}=\{\hat{u}(\zeta), \hat{w}(\zeta), \hat{p}(\zeta), 1, \hat{d}\}$, $k=k_{r}+\mathrm{i} k_{i}$ is the complex longitudinal wavenumber, $\omega=\omega_{r}+\mathrm{i} \omega_{i}$ the complex frequency, and "c.c." denotes the complex conjugate. The zero order $\left(\epsilon^{0}\right)$ basic flow corresponds to the logarithmic profile for the longitudinal velocity and the hydrostatic distribution for the pressure.

Likewise to the standard derivation of the Orr-Sommerfeld equation in hydrodynamic stability theory, ${ }^{35}$ after cross-deriving the Reynolds equations at order $\left(\epsilon^{1}\right)$, using continuity equation and the steady-state kinematic condition (i.e., $\hat{w}=\mathrm{i} k u_{0}$ at $\zeta=1$ ), we arrive at

$$
\begin{gathered}
a_{i} \mathcal{D}^{i} \hat{w}+\hat{d}=0, \\
{\left[b_{j}^{(n, t)} \mathcal{D}^{j} \hat{w}\right]_{\zeta=1}+\hat{d}=0,} \\
\left.\hat{w}\right|_{\zeta=-1}=\left[2 \hat{w}^{\prime}-\mathrm{i} k u_{0}^{\prime}\right]_{\zeta=-1}=0,
\end{gathered}
$$


where the coefficients $a_{i}(i=0,4)$ and $b_{j}^{(n, t)}(j=0,2)$ can be found in the Camporeale et al. ${ }^{36}$ superscripts ${ }^{n}$ and ${ }^{t}$ refer to the normal and tangential components of dynamic condition, respectively, and $\mathcal{D}^{i}=\partial^{i} / \partial \zeta^{i}$ (Einstein's summation notation has been adopted).

The above problem has been solved by a spectral Galerkin technique with numerical integration where boundary conditions at the free surface (3b) are imposed in weak-form, and in (3a), the fourth-order derivatives are reduced to second-order derivatives using systematic integrations by part against a suitable test function (further details can be found in the literature ${ }^{36}$ ). The solution of the flow field is therefore obtained from a discretized algebraic system and the required shear stress $\theta_{\delta}$ is substituted into the Exner equation, from which one finally arrives to the dispersion relation $D\left(k, \omega ; F, d_{s}\right)=0$. Note that the dispersion relation depends on the two control parameters $F$ and $d_{s}$.

It is well known that the space-time dynamics of an infinitesimal wavy perturbation can be investigated by the impulse response of the linearized system, namely, the Green's function. ${ }^{4}$ It reads

$$
G(x, t)=\frac{1}{(2 \pi)^{2}} \int_{\mathcal{F}} \int_{\mathcal{L}} e^{\mathrm{i}[k x-\omega t]} D^{-1}\left(k, \omega ; F, d_{s}\right) \mathrm{d} \omega \mathrm{d} k,
$$

where $\mathcal{L}$ and $\mathcal{F}$ are the Laplace and Fourier contours in the complex frequency and wavenumber planes, respectively.

In convectively unstable systems, the Green's function decays asymptotically to zero along the ray $x / t=0,{ }^{37}$ whereas it tends to infinity in an absolutely unstable system. This implies that convectively unstable systems behave as "noise amplifiers," displaying extrinsic dynamics, since in the absence of continuous forcing, the response decays back to zero, whereas absolutely unstable systems are characterized by intrinsic dynamics and behave as "oscillators." 38

By definition, the complex absolute wavenumber $k_{0}$, which represents the long time behavior of Eq. (4) at $x$ fixed, corresponds to the solution of the saddle point condition, $D(\omega, k)=\omega_{, k}(k)=0$, provided that the causality principle is satisfied, i.e., that $k_{0}$ is a pinch point. Moreover, $\omega_{0 i}$ $=\operatorname{Im}\left[\omega\left(k_{0}\right)\right]$ is the associated absolute growth rate (hereafter the subscript $i$ refers to the imaginary part). If only real wavenumbers are considered, equation $\omega_{i, k}=0$ is satisfied by the wavenumber $k_{\max }$ which displays the maximum temporal growth rate $\omega_{i, \max }=\omega_{i}\left(k_{\max }\right)$. The criteria state that the instability is convective if $\omega_{i}\left(k_{\max }\right)>0$ and $\omega_{0 i}<0$. On the contrary, the flow is absolutely unstable if $\omega_{i}\left(k_{\max }\right)>0$ and $\omega_{0 i}>0$. Recalling that the $j$ th spatial branch of the dispersion relation $k_{j}\left(\tilde{\omega}_{i}\right)$ associated with the growth rate $\tilde{\omega}_{i}$ is the locus of complex wave numbers in which $\omega_{i}(k)=\tilde{\omega}_{i}$, it can be demonstrated that points where $\omega_{, k}=0$ are pinch points only if at least two spatial branches $k_{m}\left(\omega_{0 i}\right)$ and $k_{n}\left(\omega_{0 i}\right)$ pinching in $k_{0}$ are well confined within opposite $k_{i}$ half-planes when $\omega_{i}$ is increased. ${ }^{37}$

The pinch point criteria usually require a mapping from the complex frequency plane to the complex wave number plane, thus the dispersion relation has to be solved for $k$ as a function of $\omega$. Unfortunately in many physically relevant cases (e.g., the bedform evolution herein investigated), the dispersion relation is a transcendental function of $k$ while it is polynomial only in $\omega$. In order to circumvent this difficulty, Kupfer et al. ${ }^{8}$ refined previous works ${ }^{39}$ and developed a technique, called cusp map method, for the assessment of the convective/absolute nature of the instability requiring only a $k \rightarrow \omega$ mapping.

The cusp map method basically follows two conceptual steps: (i) to detect the points that have null group velocity in the complex $\omega$-plane, and (ii) to determine whether these zero group velocity complex frequencies are actually pinch points in the complex wavenumber plane or not. The former task is accomplished recalling that (in the frequency plane) a point $\omega_{0}$ that satisfies $D\left(\omega_{0}, k\right)=D_{, k}\left(\omega_{0}, k\right)=0$ (the saddle point condition) and $D_{, k k}\left(\omega_{0}, k\right) \neq 0$, has a local map $\left(\omega-\omega_{0}\right) \sim\left(k-k_{0}\right)^{2}$. From a topological point of view, this implies that when a curve lying in the complex $k$-plane and passing through $k_{0}$ is mapped into the complex $\omega$-plane, it displays a cusp-like singular point at the branch point $\omega_{0}$. Kupfer et al. ${ }^{8}$ suggested to map the contour $k_{i}=k_{i}^{*}$, for different values of $k_{i}^{*}$, beginning from $k_{i}^{*}=0$ and then decreasing to lower values. The mapping of all contour points with $k_{i}=k_{i}^{*}$ generates in the complex frequency plane the curve $\Omega\left(k_{i}^{*}\right)$. A branch point is obtained when $\Omega\left(k_{i}^{*}\right)$ displays a cusp-like singularity (occurring at $\omega_{0}$ ), and this occurs exactly when $k_{i}^{*}=\operatorname{Im}\left(k_{0}\right)$. Finally, the check for the pinch point condition is performed by 
considering the relative position of $\omega_{0}$ with respect to $\tilde{\omega}_{F}$, the latter being the mapping of $k_{i}^{*}=0$ into the $\omega$-plane (i.e., the usual temporal branch). It can be demonstrated that branch points $\omega_{0}$ that are "covered" an odd number of times by $\tilde{\omega}_{F}$ are pinch points. ${ }^{8}$ This counting can be done by drawing a vertical line extending upward from $\omega_{0}$ and counting the number of crossing with the curve $\tilde{\omega}_{F} \cdot{ }^{40}$

Moreover, we recall that a relation between temporal and spatial theories has been derived by Gaster. ${ }^{41}$ This leads to the celebrated Gaster's transformation, valid rigorously only for a convective instability, and close to threshold. It states that, at threshold, the maximum wavenumber for the temporal theory $\left(k_{\max }\right)$ and the real part of the maximum wave number for the spatial theory are the same but that the imaginary part of the maximum wave number for the spatial theory is negative and equal to the maximum temporal growth rate $\omega_{\max }$ divided by the group velocity at $k_{\max }$. Lesser known is the fact that, under the Gaster's assumptions, the real part of the absolute complex wavenumber $k_{0 r}$ equals $k_{\max c}$, the maximum wave number for the spatial theory. This property is not generic and in particular not true away from threshold and not generic and in particular not true if the dispersion relation is more complex than the asymptotic approximation used by Gaster.

\section{RESULTS AND VALIDATION}

The results obtained by the application of the cusp-map method to the bedform instability are provided in Fig. 2 for three values of Froude numbers that are chosen to encompass both antidunes
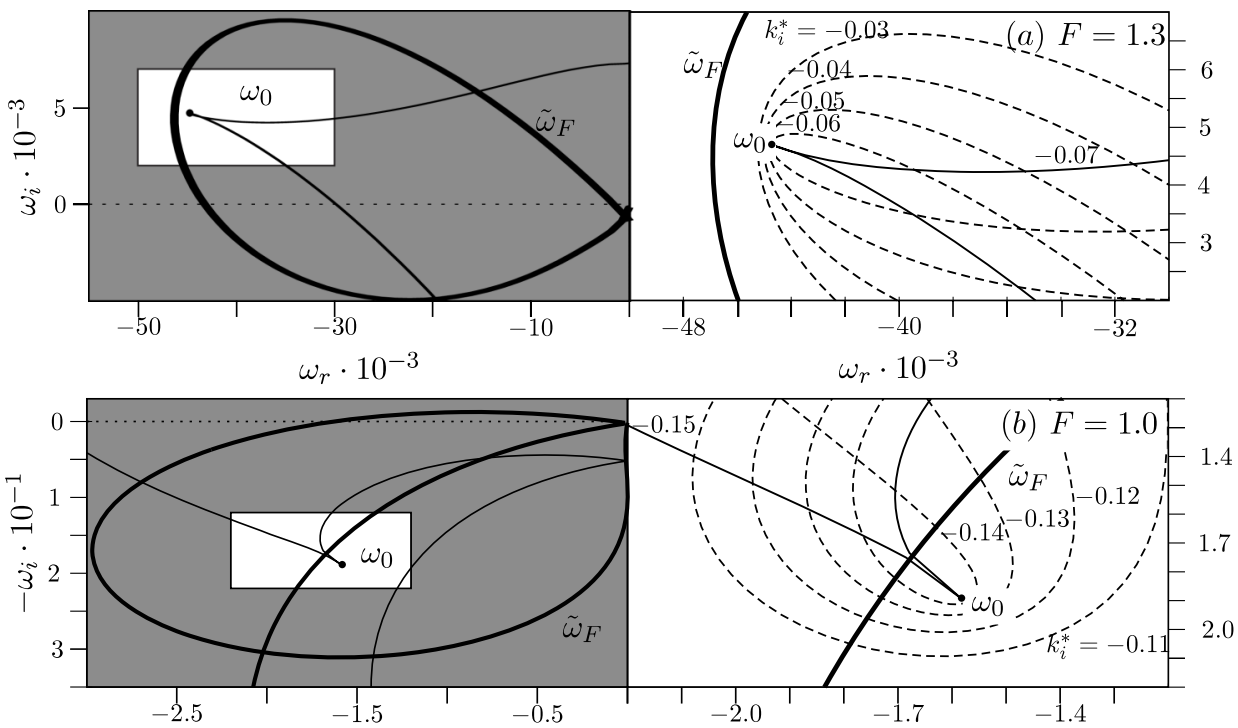

$\omega_{r} \cdot 10^{-1}$ $\omega_{r} \cdot 10^{-1}$

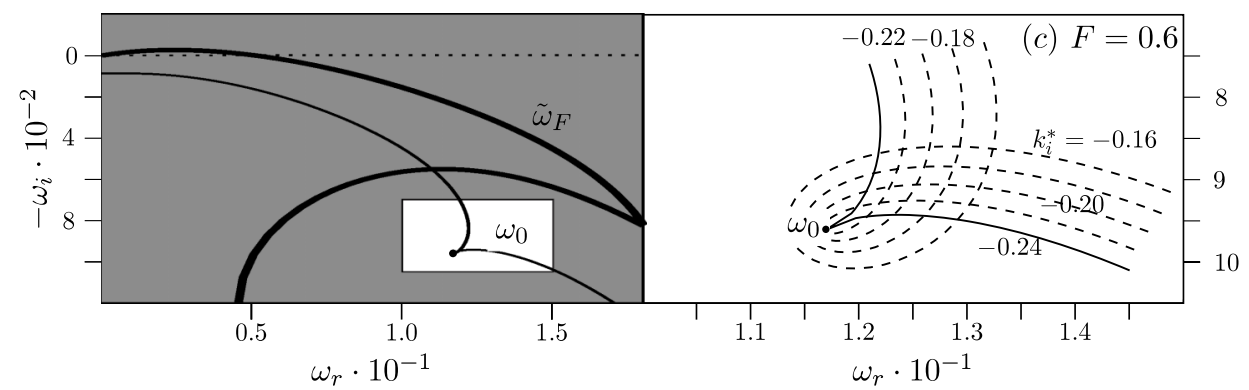

FIG. 2. Cusp maps in the $\omega$-plane for $d_{s}=1 \cdot 10^{-3}$ and three different values of $F$. The left part of the panels shows $\tilde{\omega}_{F} \equiv \Omega(0)$ and $\Omega\left(k_{0 i}\right)$, this latter displaying a branch point in $\omega_{0}$. The right part of each panels is a zoom in of the white box surrounding the branching point $\left(\tilde{\omega}_{F}\right.$ : thick solid lines; $\Omega\left(k_{0 i}\right)$ : thin solid lines; selected mappings of $\Omega\left(k_{i}^{*}\right)$ : dashed lines. The dotted lines only visible in the left panels are the $\omega_{i}=0$ line). 
(panels (a) and (b)) and dunes (panel (c)). The left part of the panels shows a plot in the $\omega$-plane of the above mentioned curve $\tilde{\omega}_{F}$ and the curve, $\Omega\left(k_{0 i}\right)$, that is the mapping of a horizontal line in $k$-plane passing through the zero-group velocity point $k_{0}$. This latter curve shows a cusp indicative of a branch point in $\omega_{0}$, whose position with respect to the contour $\tilde{\omega}_{F}$ can be studied. A white box surrounding the branch point is highlighted, corresponding to the close up plotted on the right where, along with $\tilde{\omega}_{F}$ and $\Omega\left(k_{0 i}\right)$, some mappings $\Omega\left(k_{i}^{*}\right)$ with $0>k_{i}^{*}>k_{0 i}$ are displayed. It can be observed that a reduction of $k_{i}^{*}$ from 0 to $k_{0 i}$ causes a progressive sharpening of contours $\Omega\left(k_{i}^{*}\right)$ that ultimately leads to the occurrence of the singular point at $\omega_{0}$.

At $F=1.3$, one observes that, with the lowering of the values of $k_{i}^{*}$, the contours of the mapping $\Omega\left(k_{i}^{*}\right)$ get closer to the branch point, until the curve $\Omega(-0.07)$ displays a cusp-like singularity at the branch point $\omega_{0}=(-44.72,4.69) \cdot 10^{-3}$ (see panel (a), right part). Once the branch point is detected, its position is compared with respect to $\tilde{\omega}_{F}$ and it turns out that $\omega_{0}$ is "covered" only once by $\tilde{\omega}_{F}$ since a vertical line passing through the cusp will cut only twice the $\omega_{F}$ contour (panel (a), left part). The branch point in the $\omega$-plane therefore corresponds to a pinching point in the $k$-plane. It follows that, since $\omega_{0 i}=4.69 \cdot 10^{-3}>0$, the flow is absolutely unstable.

The same procedure has been repeated for the case $F=1.0$ (panel (b)), where the branch point is detected at $k_{0 i}^{*}=-0.15$, with $\omega_{0 i}<0$. Such a branch point is not a pinch point since it is twice covered by the $\tilde{\omega}_{F}$ curve (three intersections of $\tilde{\omega}_{F}$ by a vertical line passing through $\omega_{0}$ ). Since this cusp is the first one encountered while lowering the $\mathcal{F}$ contour and it already has a negative part, the first pinch point will have an even more negative $\omega_{i}$ and the flow is therefore convectively unstable. Finally, the dune case reported in Fig. 2(c) $(F=0.6)$ displays a branch point at $\omega_{0}=(117.81,-96.13) \cdot 10^{-3}$ on the curve for $k_{0 i}^{*}=0.24$. As for the previous case, $\omega_{0}$ is covered twice by $\tilde{\omega}_{F}$, the branch point does not correspond to a pinch point, but since its $\omega_{0 i}$ is already negative, the flow is convectively unstable.

In order to check the robustness of the analysis, the sensitivity of the results reported in Fig. 2 has been studied (Fig. 3). In particular, it has been assessed how changes on the parameter $\delta$ and the introduction of the saturation length $\left(L_{S}\right)$ alter the curves $\tilde{\omega}_{F}$ (reported with thick lines) and $\Omega\left(k_{0 i}\right)$ (thin lines). The parameter $\delta$ has been altered $( \pm 20 \%)$ with respect to the value suggested
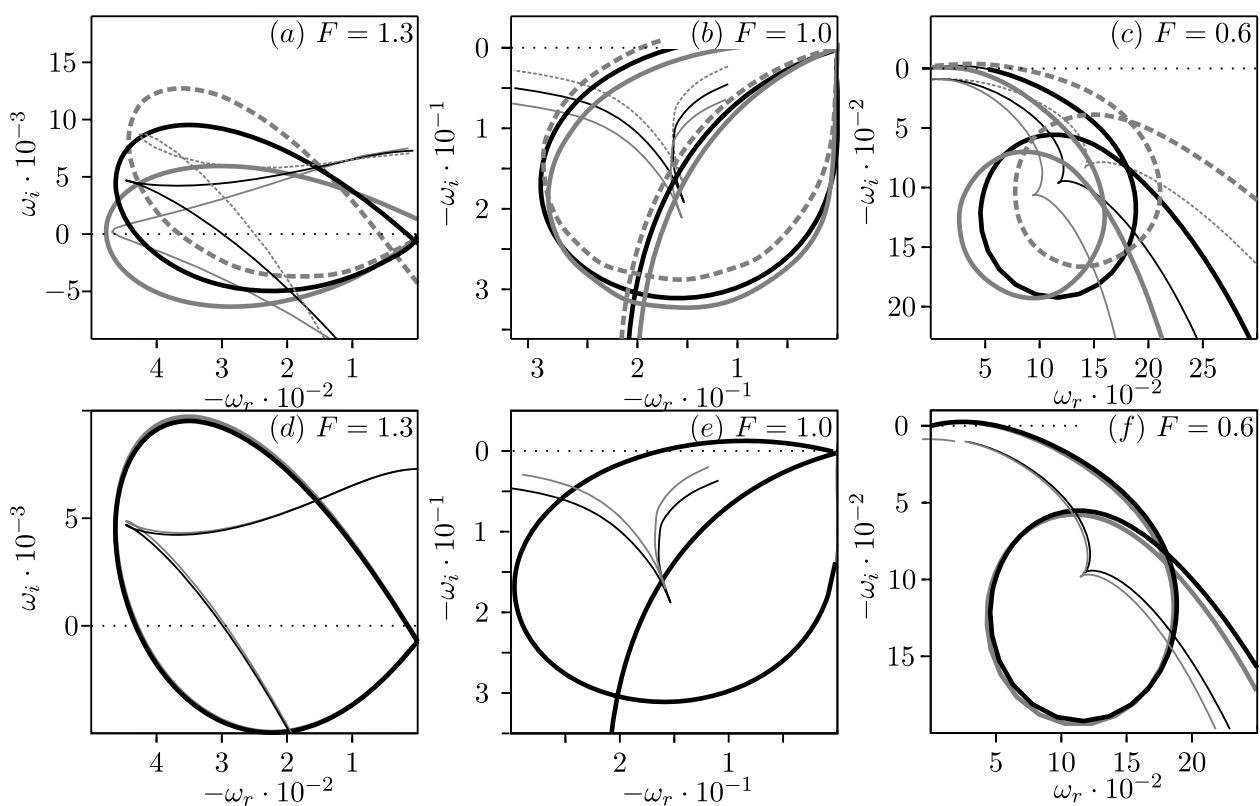

FIG. 3. Sensitivity of the curves $\tilde{\omega}_{F}$ (thick) and $\Omega\left(k_{0 i}\right)$ (thin) reported on the left panels of Fig. 2 on the main model parameters. Panels (a)-(c): effect of an increase $(+20 \%$, dashed gray lines) and of a reduction $(-20 \%$, continuous gray lines) of the parameter $\delta$ with respect to the value suggested in literature ${ }^{12}$ (continuous black lines). Panels (d)-(f): cusp maps considering the saturation length $\left(L_{s}=8 \cdot d_{s}\right.$, continuous gray lines) and not considering it ( $L_{s}=0$, continuous black lines). Curves evaluated with $L_{s}=7 \cdot d_{s}$ and $L_{s}=9 \cdot d_{s}$ are indistinguishable from the curves evaluated with $L_{s}=8 \cdot d_{s}$. 
in the literature ${ }^{12}\left(\delta=l_{b} \cdot d_{s}\right)$, and the curves evaluated with a reduced (increased) $\delta$ are drawn in continuous (dashed) gray in panels (a)-(c). A reduction (increase) of $\delta$ causes a displacement towards lower (higher) $\omega_{i}$ of both $\tilde{\omega}_{F}$ and $\Omega\left(k_{0 i}\right)$. Despite the large alteration imposed to $\delta$, the picture of the cusp maps remains substantially the same as reported in Fig. 2; for $F=1.3$, the pinch point $\omega_{0}$ remains above the line $\omega_{i}=0$ and is still covered only once by $\tilde{\omega}_{F}$ (i.e., the absolute character of the instability is preserved), while for lower $F, \operatorname{Im}\left(\omega_{0}\right)<0$, and the instability remains convective.

Second, a saturation length has been added to the sediment transport model. The curves evaluated considering $L_{s}=8 \cdot d_{s}$ (a usual value adopted by several authors ${ }^{14,20}$ for the analysis of subaqueous bedforms) are drawn in gray in panels (d)-(f). The introduction of such value has a very negligible effect (gray and black curves, that refer to $L_{s}=0$, are barely distinguishable). The analyses were repeated also for $L_{s}=7 \cdot d_{s}$ and $L_{s}=9 \cdot d_{s}$, but the curves obtained with these values overlap almost perfectly with the curves evaluated with $L_{s}=8 \cdot d_{s}$. The very small sensitivity of dune dynamics on $L_{s}$ is likely due to the different spatial scales at which saturation length occurs $\left(\sim 10 d_{s}\right)$ with respect to the typical spatial scale of bedforms $\left(\sim 10^{3} d_{s}\right)$.

An extensive and systematic analysis by the cusp-map method over the $\left(d_{s}, F\right)$ parameter space is reported in Fig. 4, in the ranges $d_{s}=0.006-0.01$ and $F=0.16-1.8$. This analysis provides the main result of the present study. In the region where $\omega_{0 i}>0$, one needs to check whether the causality principle is satisfied, namely, whether every branch point associated with the cusp singularity is a pinch point. Five different zones emerge in the parameter space: (i) absence of the sediment transport $\left(\theta_{\delta}<\theta_{c}\right.$, hatched zone); (ii) stable flat bed conditions ( $\omega_{i, \max }<0$, white zone); (iii) convective downstream migrating instabilities (i.e., the phase velocity is positive, namely, $\omega_{0 r}>0$ ), here named convective dunes (CD); (iv) convective upstream migrating instabilities (negative phase velocity, corresponding here to $\omega_{0 r}<0$ ), named convective antidunes (CA); (v) absolute upstream migrating instabilities, named absolute antidunes (AA). Moreover, the contours $\theta=0.4$ (dotted) and $\theta=1$ (dot-dash) are reported. These two values are the lower and higher thresholds reported in the literature above which the inception of sheet-flows is observed. ${ }^{42}$ In the occurrence of sheet flows, the mathematical model adopted in this analysis no longer represents all physical process involved in the sediment transport, and caution should be used in interpreting the results of the present analysis in the case of both very fine sediments and very high Froude number. It appears that dune patterns are invariably convectively unstable, whereas antidunes can be either absolutely or convectively unstable,

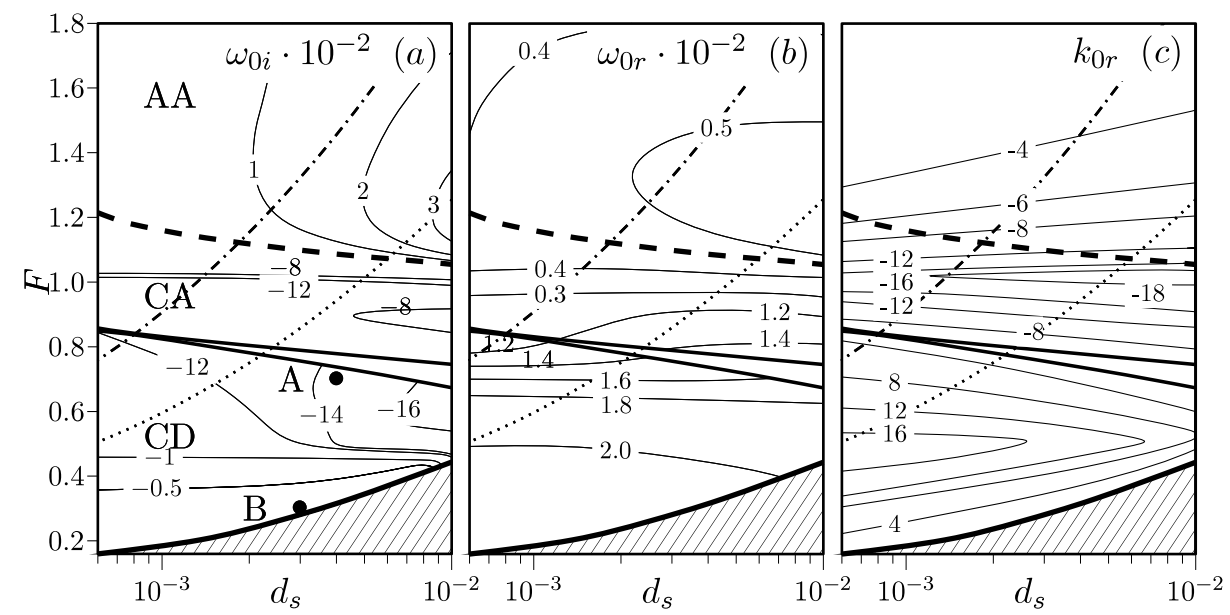

FIG. 4. (a) Contour map of the absolute growth rate, $\omega_{0 i}$, (b) the absolute frequency, $\omega_{0 r}$, (c) the absolute wave number, $k_{0 r}$, by the cusp map method as a function of the sediment diameter and Froude number. Hatched zone indicates no sediment transport (points where $\left.\theta<\theta_{c} \sim 0.05\right)$. The dotted (dotted-dashed) line reports $\theta=0.4(\theta=1.0)$. Thick black lines separate stable from unstable regions. A dashed line highlights $\omega_{0 i}=0$ and separates AA from CA. Point A $\left(d_{s}=0.0043\right.$, $F=0.7)$ and point $\mathrm{B}\left(d_{s}=0.0032, F=0.29\right)$ mark the parameters used in the numerical simulation by Giri et al $^{25}$ and in the experiments of Venditti et al..$^{24}$ respectively. 
slightly depending on the relative roughness, $d_{s}$, but to a great extent on the Froude number. Note that in some regions of the $\mathrm{CA}$ domain and in the whole $\mathrm{CD}$ domain, the reported branch point and the associated value of $\omega_{0}$ and $k_{0}$ are meaningless as already explained. Fig. 4 also shows the absolute frequency and the absolute wave number. They only matter in the absolute domain AA where they predict the linear instability that should appear in the absence of any continuous forcing. The absolute frequency at the convective/absolute threshold is negative, since the antidunes lead the transition, and its magnitude increases from 0.06 at $d_{s}=10^{-3}$ to 0.14 at $d_{s} \sim 10^{-2}$ and decreases for larger values. On the contrary, the absolute wavenumber at threshold stays nearly constant at about 0.4 (Fig. 4(c)).

The above theoretical analysis is compared with some numerical and experimental works of the existing literature on dune formation. In the paper by Giri and Shimizu ${ }^{25}$ a numerical code for the computation of sand dune migration was developed and tested against a large amount of experimental data, thus demonstrating that simulations are able to reproduce some key features, such as velocity profiles, turbulence characteristics, dune migration, and amalgamation processes. Stream dynamics was modeled through Reynolds Averaged Navier-Stokes equations, the Reynolds stresses being evaluated by a nonlinear $k-\epsilon$ turbulence closure model. The morphological evolution of the bottom was described by deposition/pick-up particle rates, evaluated through an Eulerian stochastic approach. ${ }^{43}$ Simulations were performed in a rectangular computational domain, where a flat bed with very small random perturbations was set as initial conditions. A summary of the outcomes of a run by Giri and Shimizu ${ }^{25}$ set at the conditions of point A of Fig. 4, are reported in (Figs. $5(\mathrm{a})-(\mathrm{c}))$. Downstream to the point $x^{*}=60 \mathrm{~cm}$, the first perturbations in the domain become visible at $t=280 \mathrm{~s}$. At $t=349 \mathrm{~s}$, dune patterns are present in the region $x=75-140 \mathrm{~cm}$, while upstream the perturbation has completely reverted to a flat bed. This behavior continues forward in time and, at $t=490 \mathrm{~s}$ (panel (c)), flat bed recovery stretches out on the region $x^{*}<95 \mathrm{~cm}$. Numerical simulations therefore show that the bed perturbations decay to zero with time, since they are convected out of the domain; in other words, the instability has been observed to be convective, in agreement with our theoretical prediction (see Fig. 4), since it grows in amplitude but is convected away.

Another set of observations to which we may compare has been provided by Venditti et al., ${ }^{24}$ which developed an experimental work on the onset of sandy bedforms. The experiments were conducted in a $15.2 \mathrm{~m}$ long and $1 \mathrm{~m}$ wide flume, with the median grain size $d_{s}^{*}=0.5 \mathrm{~mm}$. The initial flat bed was artificially perturbed to allow dune formation and the bed evolution was detected by an eco-sounder. An illustrative case, adapted from their results, is reported in (Figs. 5(d)-(f)) for the conditions represented by point B in Fig. 4. Both positive and negative initial perturbations (conical sand heap or hole, respectively) were considered. The response to the positive perturbation is shown by a lateral longitudinal section reported in panels (Figs. 5(d)-(f)). In this case, after one hour, the perturbation has significantly grown and spread downstream (till $x^{*} \sim 100 \mathrm{~cm}$ ) but, apart
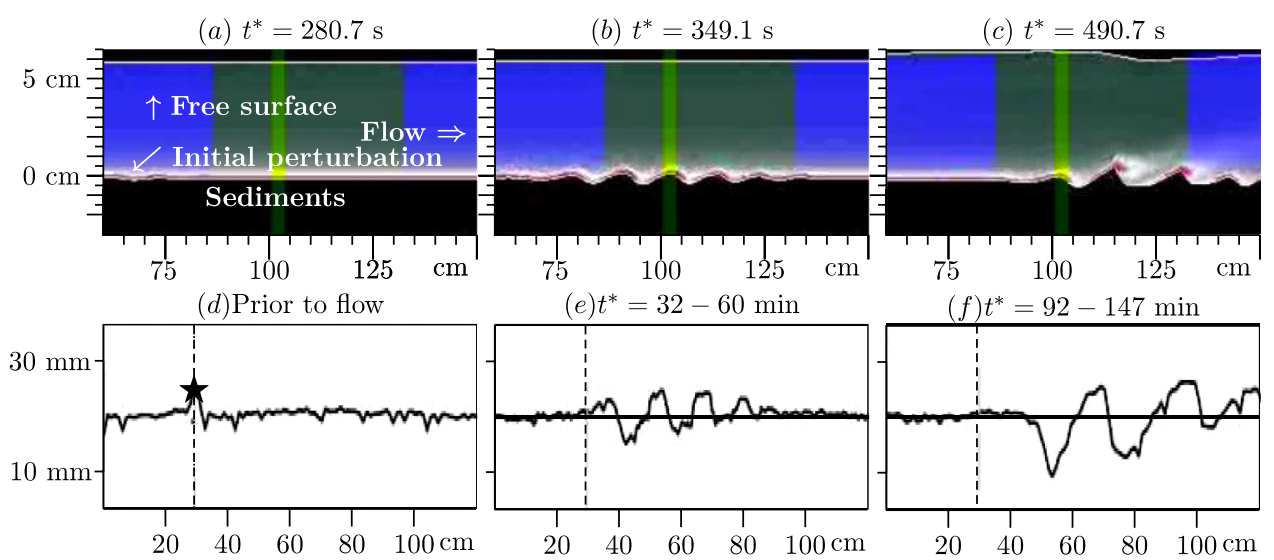

FIG. 5. (a)-(c) Results of a 2D numerical simulation by Giri and Shimizu ${ }^{25}$ performed at $d_{s}=0.0043$ and $F=0.70$, adapted from the original figure. (d)-(f) Flume experiments by Venditti et al. ${ }^{24}$ Notice (both in panels (a)-(c) and (d)-(f)) that the horizontal scale is several times lower than the vertical scale, and bedforms have a wavelength to depth ratio of about 3-4, corresponding to dunes instability. 
from random noise comparable to $d_{s}^{*}$, the perturbation has relaxed back to the flat bed at locus of the localized initial deformation $\left(x^{*}=30\right)$. In the next frame, after 147 min (panel (f)), the amplitude has kept increasing downstream whereas a complete recovery of a flat bed occurs at $x^{*}<40 \mathrm{~cm}$. These flume experiments confirm dunes to be convectively unstable, as predicted by the presented theoretical analysis.

\section{CONCLUDING REMARKS}

We have demonstrated that river dunes are due to a convective morphological instability, while antidunes are due to an instability which may be either convective or absolute, depending on the physical control parameters. The absolute antidune instability occurs for Froude numbers above a threshold value that varies weakly with the grain size ratio, $d_{s}$, from $F_{A}=1.2$ at $d_{s}=10^{-3}$ to $F_{A}$ reaching unity at $d_{s}=10^{-1}$. For the dune regime, the present prediction is consistent with the numerical results of Giri and Shimizu ${ }^{25}$ and with the experimental results of Venditti et al. ${ }^{24}$ For antidunes, the possibility of a transition from convective to absolute instability, while increasing either $F$ or $d_{s}$, is calling for experimental or numerical validation. These findings also play an important role in the interpretation of flume or field experiments. For example, in laboratory, experiments run in the parameter range where either dunes or antidunes are convectively unstable (domains $\mathrm{CD}$ and CA in Fig. 4), one has to take into account the fact that experiments are highly affected by flume imperfections, as the sediment bed behaves like a noise amplifier; similarly, actual river bed forms should be interpreted as the result of the spatial development and nonlinear evolution of instabilities forced by the upstream perturbation. On the contrary, in the AA domain, the dynamics should be the intrinsic result of the nonlinear evolution of an absolute instability that should prevail over the noise, allowing the experimental result to be less dependent on the facility. In numerical simulations these aspects have to be considered as well; the common trick of a periodic setting of the boundary conditions is unable to reproduce the dynamics neither of convective nor of absolute instability. As a matter of fact, only when the flow is strongly convective (i.e., $\omega_{0 i} \ll 0$ ) or the instability incipient, the temporal growth observed in periodic domain can be transformed in a spatial growth for the real flow by the Gaster's ${ }^{41}$ transformation. This transformation states that the maximum wavenumber for the temporal theory $\left(k_{\max }\right)$ and the real part of complex wave number $\left(k_{\max }^{C}\right)$ with the largest spatial growth rate for the spatial theory are the same, whereas the imaginary part of this spatial wavenumber is related to the maximum temporal growth rate through $k_{i, \max }^{C}=-\omega_{i, \max } /\left(d \omega /\left.d k\right|_{k_{\max }}\right)$.

We stress that the analyses here performed are based on the Meyer-Peter-Müller closure relation for bedload transport. In some previous tests, we have verified that the growth rate and the celerity of the bedforms are very little influenced by the change of the sediment transport closure relation. In particular, the absolute values of the growth rate and the celerity change in a very narrow range and, more importantly, their sign is never altered. This means that, at linear level, the bedforms dynamics is mainly driven by the stream dynamics, and the results presented here are valid for any bedload transport relationship.

We also recall (Fig. 4) that $d_{s}$ does not play a major role in determining the absolute/convective nature of the bedforms instability. We therefore expect that bedforms in heterogeneous sediments exhibit instabilities in accordance to the results reported here.

Summing up, the marginal role played by the sediment transport closure relationship and by the sediment heterogeneity implies that the results here described are valid for real rivers; dunes are expected to exhibit a convective behavior and antidunes to exhibit an absolutely unstable behavior for stream Froude number exceeding the threshold of about 1.1.

It is also worth to revisit the results from paleo-hydraulic reconstructions. This technique has been successfully used in the past ${ }^{7,44,45}$ for the estimation of the flow rate from the morphology of bedforms found in rock records. This work does not pretend to directly address this issue, but the findings here reported can have an important implication in this context. In fact, convective instabilities behave like noise amplifiers, regardless of the role of nonlinearities ${ }^{4}$ and therefore their response is strongly affected by initial conditions. It means that convective bedforms exhibit 
different wavelength under the same flow. On the contrary, absolute instabilities behave like oscillators and their response is less affected by initial conditions, as they impose their intrinsic dynamics. It means that absolute antidunes will exhibit the same wavelength under the same flow. It follows that paleohydraulic reconstructions are more robust for absolute instabilities than for convective ones. This is likely the reason why all reported cases of paleohydraulic reconstruction to our knowledge were performed by analyzing antidune patterns.

Our findings can also be crucial in river engineering, especially where bedforms interact with infrastructures or navigation. As a matter of fact, we have demonstrated that convective instabilities of the bed are triggered and are controlled by upstream disturbances. Therefore, the reduction of the amplitude of the disturbance that triggers the bedform pattern can result in a mitigation of bed disturbances. We stress that this approach is limited by the strong spatial amplification of the upstream disturbances. Finally, the transition from convective to absolute antidunes (by increasing $F$ or $d_{s}$ ) can play an important role in the understanding of complex spatial morphological patterns occurring in rivers under non-uniform flow conditions. Non-uniform conditions are very frequent, e.g., due to bed slope changes, gates, etc., and their interplay with antidunes can significantly affect the global stability of the system. ${ }^{4,46}$

${ }^{1}$ R. Vesipa, C. Camporeale, and L. Ridolfi, “Transient growths of stable modes in riverbed dynamics,” EPL 100, 64002 (2012).

${ }^{2}$ M. Colombini and A. Stocchino, "Three-dimensional river bed forms," J. Fluid Mech. 695, 63-80 (2012).

${ }^{3}$ F. Nunez-Gonzalez and J. Martin-Vide, "Analysis of antidune migration direction," J. Geophys. Res. 116, doi: 10.1029/2010JF001761 (published online 2011).

${ }^{4}$ P. Huerre and P. A. Monkevitz, "Local and global instabilities in spatially developing flows," Annu. Rev. Fluid Mech. 22, 473-537 (1990).

${ }^{5}$ B. Federici and G. Seminara, "On the convective nature of bar instability," J. Fluid Mech. 487, 125-145 (2003).

${ }^{6}$ C. Camporeale and L. Ridolfi, "Convective nature of the planimetric instability in meandering river dynamics," Phys. Rev. E 73, 026311 (2006).

${ }^{7}$ J. Shaw and R. Kellerhals, "Paleohydraulic interpretation of antidune bedforms with applications to antidunes in gravel," J. Sediment. Petrol. 47, 57-266 (1977).

${ }^{8}$ K. Kupfer, A. Bers, and A. K. Ram, "The cusp map in the complex-frequency plane for absolute instabilities," Phys. Fluids 30, 3075-3082 (1987).

9 J. F. Kennedy, "The mechanics of dunes and antidunes in erodible-bed channels," J. Fluid Mech. 16, 521-544 (1963).

${ }^{10}$ K. J. Richards, "The formation of ripples and dunes on an erodible bed," J. Fluid Mech. 99, 597-618 (1980).

${ }^{11}$ S. E. Coleman and J. D. Fenton, "Potential-flow instability theory and alluvial stream bed forms," J. Fluid Mech. 418, 101-117 (2000).

12 M. Colombini, "Revisiting the linear theory of sand dunes," J. Fluid Mech. 502, 1-16 (2004).

${ }^{13} \mathrm{~J}$. Best, "The fluid dynamics of river dunes: A review and some future research directions," J. Geophys. Res. 110, doi: 10.1029/2004JF000218 (published online 2005).

${ }^{14}$ A. Fourriere, P. Claudin, and B. Andreotti, "Bedforms in a turbulent stream: Formation of ripples by primary linear instability and of dunes by nonlinear pattern coarsening," J. Fluid Mech. 649, 287-328 (2010).

${ }^{15}$ G. Seminara, "Fluvial sedimentary patterns," Annu. Rev. Fluid Mech. 42, 43-66 (2010).

${ }^{16}$ E. Lajeunesse, L. Malverti, P. Lancien, F. Metivier, S. Coleman, C. E. Smith, T. Davies, A. Cantelli, and G. Parker, "Fluvial and submarine morphodynamics of laminar and near-laminar flows: A synthesis," Sedimentology 57(1), 1-26 (2010).

${ }^{17}$ M. Colombini and A. Stocchino, "Ripple and dune formation in rivers," J. Fluid Mech. 673, 121-131 (2011).

${ }^{18}$ G. Seminara, L. Solari, and G. Parker, "Bed load at low shields stress on arbitrarily sloping beds: Failure of the bagnold hypothesis," Water Resour. Res. 38(11), 1249 (2002).

${ }^{19}$ G. Parker, G. Seminara, and L. Solari, "Bed load at low shields stress on arbitrarily sloping beds: Alternative entrainment formulation,” Water Resour. Res. 39(7), 1183 (2003).

${ }^{20}$ F. Charru, B. Andreotti, and P. Claudin, "Sand ripples and dunes," Annu. Rev. Fluid Mech. 45, 469-493 (2013).

${ }^{21}$ C. Camporeale and L. Ridolfi, "Modal versus nonmodal linear stability analysis of river dunes," Phys. Fluids 23, 104102 (2011).

${ }^{22}$ H. P. Guy, D. B. Simons, and E. V. Richardson, "Summary of alluvial channel data from flume experiments," U.S. Geol. Surv. Prof. Pap. 462-I, 1-96 (1966).

${ }^{23}$ M. Colombini and A. Stocchino, "Finite amplitude river dunes," J. Fluid Mech. 611, 283-306 (2008).

${ }^{24}$ J. G. Venditti, M. A. Church, and S. J. Bennett, "Bed form initiation from a flat sand bed," J. Geophys. Res. 110, doi: 10.1029/2004JF000149 (published online 2005).

${ }^{25}$ S. Giri and Y. Shimizu, "Numerical computation of sand dune migration with free surface flow," Water Resour. Res. 42 (2006).

${ }^{26}$ S. E. Coleman and B. W. Melville, "Initiation of bed forms on a flat sand bed," J. Hydraul. Div., Am. Soc. Civ. Eng. 122, 301-310 (1996).

${ }^{27}$ J. G. Venditti, M. Church, and S. J. Bennett, “On the transition between 2d and 3d dunes," Sedimentology 52, 1343-1359 (2005).

${ }^{28}$ S. Pope, Turbulent Flows, 1st ed. (Cambridge University Press, 2000). 
${ }^{29}$ I. Nezu and W. Rodi, “Open-channel flow measurements with a laser doppler anemometer,” J. Hydraul. Eng.-ASCE 112(5), 335-355 (2005).

${ }^{30}$ F. M. Exner, Uber die Wechselwirkung zwischen Wasser und Geschiebe in Flussen, 1st ed. (Sitzber. Akad. Wiss Wien, 1925).

31 J. Fredsoe, "On the development of dunes in erodible channels," J. Fluid Mech. 64, 1-16 (1974).

${ }^{32}$ G. Parker, "Sediment inertia as cause of river antidunes," J. Hydraul. Div.-ASCE 101(2), 211-221 (1975).

${ }^{33}$ M. Sekine and H. Kikkawa, "Mechanics of saltating grains," J. Hydraul. Div., Am. Soc. Civ. Eng. 118, 536-558 (1992).

${ }^{34}$ H. Y. Lee and I. S. Hsu, "Investigation of saltating particle motion," J. Hydraul. Eng. 120, 831-845 (1994).

${ }^{35}$ P. Schmid and D. Henningson, Stability and Transition in Shear Flows, 1st ed. (Springer, 2001).

${ }^{36}$ C. Camporeale, C. Canuto, and L. Ridolfi, "A spectral approach for the stability analysis of turbulent open-channel flows over granular beds," Theor. Comput. Fluid Dyn. 26, 51-80 (2012).

${ }^{37}$ A. Bers, "Space-time evolution of plasma instabilities - absolute and convective," in Handbook of Plasma Physics (North Holland, Amsterdam, 1983).

${ }^{38}$ P. Huerre and M. Rossi, "Hydrodynamic instabilities in open flows," in Hydrodynamic and Instabilities, edited by C. Goldreche and P. Manneville (Cambridge University Press, Cambridge, 2000), pp. 159-229.

${ }^{39}$ H. Derfler, "Frequency cusp, a menas for discriminating between convective and nonconvectibe instability," Phys. Rev. A 1, 1467-1471 (1970).

${ }^{40}$ Q. D. Zhang, B. C. Khoo, and K. S. Yeo, "A numerical study of the effect of free surface and water depth on the stability of wakes: Use of gdq formulation,” Int. J. Numer. Methods Fluids 24, 1079-1090 (1997).

${ }^{41} \mathrm{M}$. Gaster, "A note on the relation between temporally-increasing and spatially-increasing disturbances in hydrodynamic stability," J. Fluid Mech. 14, 222-224 (1962).

${ }^{42}$ P. Gao, "Transition between two bed-load transport regimes: Saltation and sheet flow,” J. Hydraul. Div., Am. Soc. Civ. Eng. 134, 340-349 (2008).

${ }^{43}$ H. Nakagawa and T. Tsujimoto, "Sand bed instability due to bed load motion," J. Hydraul. Div., Am. Soc. Civ. Eng. 106, 2029-205 (1980).

44 G. Middleton, “Antidune cross-bedding in a large flume,” J. Sediment. Res. 35, 922-927 (1965).

${ }^{45}$ B. Rust and M. Gibling, "3-Dimensional antidunes as HCS mimics in a fluvial sandstone - the Pennsylvanian South bar formation near Sydney, Nova-Scotia,” J. Sediment. Petrol. 60, 540-548 (1990).

46 J. M. Chomaz, "Global instabilities in spatially developing flows: Non-normality and nonlinearity," Annu. Rev. Fluid Mech. 37, 357-392 (2005). 